\title{
RECUPERACIÓN DEL FUNCIONAMIENTO HIDROLÓGICO DE ECOSISTEMAS DE PÁRAMO EN COLOMBIA
}

\section{REHABILITATION OF HYDROLOGICAL FUNCTIONING OF PÁRAMO ECOSYSTEMS IN COLOMBIA}

\author{
María Fernanda Cárdenas ${ }^{1}$, Conrado Tobón ${ }^{2}$
}

\begin{abstract}
${ }^{1}$ Ingeniera Forestal, Esp. y M.Sc. en Planeación Urbana - Regional, Ph.D en Ingeniería - Recursos Hidráulicos. Universidad Nacional de Colombia, Medellín, Calle 56 N78A-59. Correo electrónico: mfcarden@unal.edu.co; ${ }^{2}$ Agrólogo, M.Sc. en física de suelos, Ph.D en biodiversidad y ecosistemas dinámicos. Profesor Titular, Departamento de Ciencias Forestales Universidad Nacional de Colombia, Medellín. Calle 59A Nº3-20. Correo electrónico: ctobonm@unal.edu.co
\end{abstract}

Rev. U.D.C.A Act. \& Div. Cient. 20(2): 403-412, Julio- Diciembre, 2017

\section{RESUMEN}

Los páramos son ecosistemas que se caracterizan por una alta humedad relativa, alta frecuencia de niebla y por tener una vegetación arbustiva y herbácea, adaptada a las condiciones de alta montaña. En condiciones naturales estos ecosistemas presentan una oferta hídrica alta, entre otros servicios ecosistémicos; sin embargo, esto cambia cuando son degradados. A pesar de su importancia son ecosistemas que han sido modificados por el hombre, la mayoría aún sin haber sido estudiados. Este documento presenta los resultados de una investigación realizada en tres páramos en Colombia, en la cual, se evalúa su funcionamiento hidrológico y la capacidad de recuperación, una vez han sido alterados; para esto, se estudió el clima, la hidrología y los suelos. Los resultados indican que estos ecosistemas tienen condiciones climáticas desiguales, que determinan su rendimiento y regulación hídrica; con diferencias en las propiedades hidrofísicas del suelo y contenido de materia orgánica, lo que determina diferencias en su capacidad para retener la humedad y la disponibilidad de agua para las plantas. Estos resultados indican que los páramos, con un grado bajo de disturbio, presentan un alto rendimiento y buena regulación hídrica; pero igual que los páramos intervenidos, recuperan el estado natural de las propiedades del suelo y, consecuentemente, su funcionamiento hidrológico, aún en pocas décadas.

Palabras clave: Ecosistemas de alta montaña tropical, degradación, propiedades hidro-físicas, suelos de páramo, rendimiento hídrico, regulación hídrica.

\section{SUMMARY}

Páramos are equatorial alpine ecosystems characterized by a high air humidity, frequency of fog and the presence of shrub and herbaceous vegetation adapted to the specific equatorial alpine conditions. Under natural conditions these ecosystems present a high water yield, among other ecosystem services, however, this changes when they are degraded. Despite their importance, they are ecosystems that have been modified by man, most of them even without having been studied. This paper presents the results of a hydrological research carried out in three páramos in Colombia, which evaluates their hydrological functioning and their capacity for recovery once they are altered. Therefore, we studied their climate, hydrology, and soils properties. Results indicate that these ecosystems have different climatic conditions, which determine their water yield and water regulation. Differences in the hydro-physical properties of soils and organic matter content, which determine differences in their capacity to retain water and on the magnitude of the water available for plants. These results indicate that undisturbed páramos or with a low degree of disturbance present high water yield and good water regulation; and intervened páramos can recover the natural conditions of soil properties, and consequently their hydrological functioning, even in a few decades.

Key words: Equatorial alpine ecosystems, degradation, hydro-physical soil properties, páramo soils, water yield, water regulation.

\section{INTRODUCCIÓN}

A nivel global, se reconoce que las montañas albergan ecosistemas diversos, frágiles y muy valiosos, tanto por su biodiversidad como por su abundancia en agua (Becker, 2005). En particular, los sistemas montañosos son elementos clave en el ciclo hidrológico y están vinculados con el soporte de la biota, en la mayoría de las regiones del mundo (Diaz et al. 2003). 
En la alta montaña tropical, los páramos se reconocen como unos de los ecosistemas que juegan un papel fundamental en la regulación hidrológica y en la provisión de agua para la población (Buytaert et al. 2006a), así como otra gran variedad de servicios ambientales asociados (Mosquera et al. 2015).

Los páramos, se localizan, aproximadamente, entre los $11^{\circ} \mathrm{N}$ y $8^{\circ} \mathrm{S}$ de latitud, distribuidos como parches discontinuos en las partes más altas de las montañas, en zonas demasiado frías, para el desarrollo de bosques (Guzmán et al. 2015; Hofstede et al. 2003) y consisten en formaciones accidentadas, muchas de ellas, de origen glaciar, que contienen una variedad de lagos, humedales y pantanos (Buytaert et al. 2006a), con una vegetación en la que predominan los pajonales, mezclados con arbustos y con rosetas gigantes.

De acuerdo con Ruiz et al. (2008), las condiciones climáticas promedio de estos ecosistemas, se caracterizan por temperaturas bajas con un amplio rango diurno, alta nubosidad y presencia continua de niebla y lloviznas, baja presión atmosférica y vientos fuertes. Otra de las características de los páramos son sus suelos ricos en materia orgánica y, en general, con excelentes propiedades biofísicas (Buytaert et al. 2006c; Farley et al. 2004), que explican la gran capacidad que tienen para almacenar y retener agua (Buytaert et al. 2006b).

A pesar de tener asociados importantes servicios ecosistémicos, como la regulación y la provisión de agua, el almacenamiento de carbono y la biodiversidad, los páramos y su gestión afrontan dos problemas principales: i) escaso conocimiento acerca de su funcionamiento ecohidrológico (Goldsmith et al. 2012; Buytaert et al. 2006a; Célleri \& Feyen, 2009; Mosquera et al. 2015), que es más grave, si se considera el amplio gradiente espacio-temporal y la variabilidad en las condiciones hidro-meteorológicas que presentan estos ecosistemas (Ochoa-Tocachi et al. 2016), a lo cual, se puede sumar la incertidumbre por los efectos que el cambio climático pueda tener sobre su estructura y funcionamiento y, ii) las intervenciones y la presión antrópica para desarrollar actividades productivas sobre áreas cada vez de mayor altitud, expresadas como el avance de la frontera agropecuaria, que ha venido ocurriendo en la mayoría de los páramos Andinos (Hofstede et al. 2003), ocasionando la destrucción de la vegetación natural, erosión y deterioro de las propiedades de los suelos. Lo anterior puede resultar en la disminución de la capacidad de retención de humedad y de la calidad del agua superficial e infiltrada, al igual que en el incremento en la meteorización de la materia orgánica y en la pérdida de nutrientes (Morales et al. 2007). Adicionalmente, la vulnerabilidad de los ecosistemas de alta montaña tropical ante el cambio climático ha sido estudiada desde diversas aproximaciones y, en general, señalan que sus características los hacen ecosistemas vulnerables (Herzog et al. 2012).
Con el fin de entender mejor el funcionamiento y la capacidad de recuperación de las características ecohidrológicas de los ecosistemas de páramo, luego de intervenciones humanas, en este documento, se presentan los resultados de una investigación llevada a cabo en tres páramos en Colombia, donde se evaluaron diferentes variables climáticas, hidrológicas y de suelos, teniendo en cuenta la historia de intervenciones o el nivel de protección que cada uno posee.

\section{MATERIALES Y MÉTODOS}

Sitios de estudio. La presente investigación, se llevó a cabo en tres páramos: el páramo de Belmira (Antioquia, cuenca sin nombre), el páramo de Chingaza (Cundinamarca, cuenca Calostros) y el páramo de Romerales (Quindío, cuenca Cárdenas). Belmira y Romerales, se sitúan en la Cordillera Central de Colombia y Chingaza está localizado en la Cordillera Oriental.

El páramo de Belmira tiene suelos derivados de rocas ígneas y metamórficas, parcialmente cubiertos de cenizas volcánicas meteorizadas. En el pasado estuvo sometido a ganadería extensiva y a minería de oro, lo que resultó en una notable degradación del suelo, pero mediante el Decreto 1974 de 1989 fue declarado Distrito de Manejo Integrado de los Recursos Naturales Renovables (Corantioquia, 1999) y se encuentra bajo un programa de protección, ya que está asociado con el abastecimiento de agua para el Área Metropolitana del Valle de Aburrá.

El páramo de Chingaza hace parte de un gran complejo ubicado en la Cordillera Oriental, desarrollado a partir de movimientos de masas glaciares, areniscas y pizarras; son suelos con alto contenido de materia orgánica y sin cenizas volcánicas. Se encuentra en un estado de conservación óptimo, es uno de los ecosistemas asociados al abastecimiento de agua para Bogotá y está protegido dentro del Sistema de Parques Naturales Nacionales (PNN) de Colombia, desde 1977 (LoraGómez, 2005).

El páramo de Romerales, se encuentra en el macizo volcánico de la Cordillera Central, en la zona de influencia del PNN Los Nevados. Sus suelos están desarrollados a partir de materiales volcánicos medianamente meteorizados. Este sistema ha estado sometido por décadas a cultivos y a ganadería extensiva, por lo cual, hoy en día, evidencia deterioro y erosión de sus suelos (Salento, 2000).

En cada páramo, se seleccionó una cuenca hidrográfica que fuera lo más representativa posible del ecosistema en su conjunto. La figura 1 presenta la localización de los páramos estudiados, las coberturas presentes y la ubicación aproximada de los equipos instalados en cada una de las cuencas y de los sitios seleccionados para muestrear los suelos. 
a)

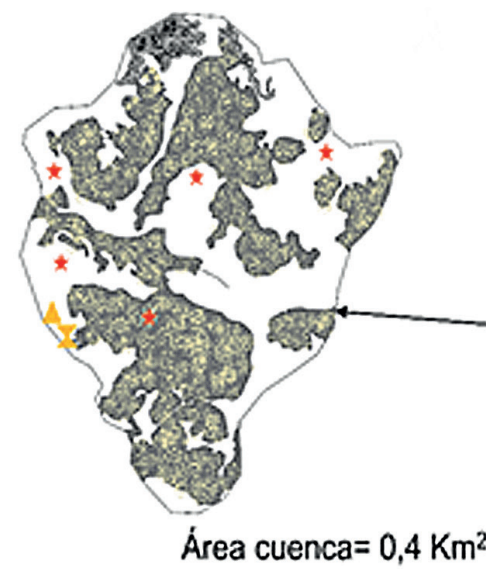

c)

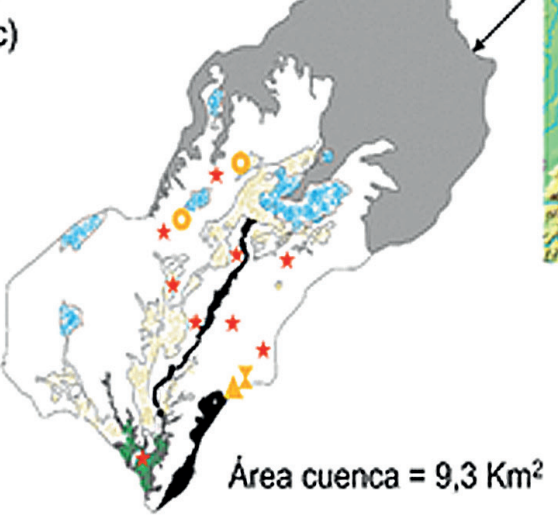

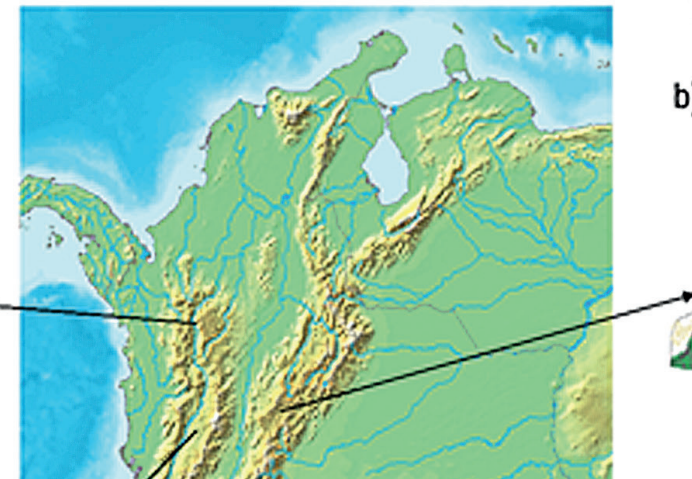

19.

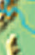

b)

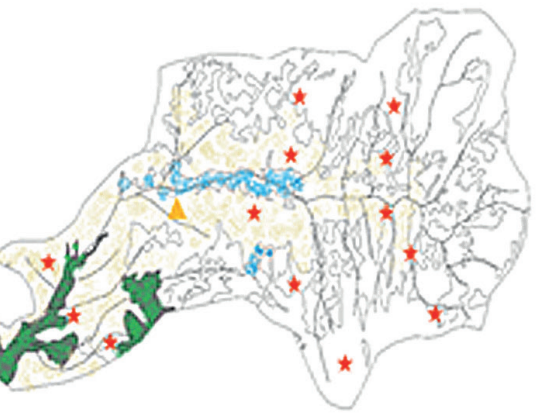

Área cuenca $=7,5 \mathrm{Km}^{2}$

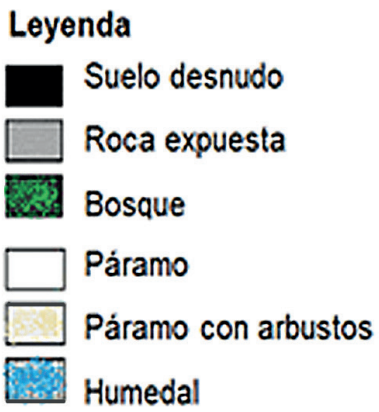

Figura 1. Localización de los sitios de estudio en Colombia; extensión, coberturas, ubicación de los instrumentos y sitios de muestreo de suelos, en cada una de las cuencas seleccionadas en los páramos de: a) Belmira, b) Chingaza y c) Romerales.

Medición de variables hidro-meteorológicas. En cada sitio, se midieron las variables precipitación, temperatura, humedad del aire, radiación solar, velocidad y dirección del viento, durante dos años (2014 y 2015), cada 15 minutos, usando estaciones meteorológicas (Davis y Campbell Sci). Asimismo, se instalaron un conjunto de pluviómetros (Texas Instr.), con registradores (Tynitag), que guardaron datos cada $5 \mathrm{mi}-$ nutos, distribuidos en las cuencas, con el fin de determinar, además, la distribución espacial de la precipitación. En los puntos de cierre de cada cuenca, se midieron los caudales usando canales mejorados, a modo de vertederos rectangulares, dotados con un limnímetro y un barómetro (Solinst); estos últimos guardaban datos cada 5 minutos.

Propiedades hidro-físicas del suelo. Las propiedades físicas e hidrológicas de los suelos también se evaluaron en los tres páramos. Para ello, se seleccionaron los lugares de muestreo, teniendo en cuenta la heterogeneidad del territorio, de modo que el conjunto fuera representativo de las con- diciones de las cuencas. En Belmira, se muestrearon 5 sitios, en Chingaza 12 y en Romerales 9. En cada sitio de muestreo se hizo una calicata, incluyendo el horizonte C; se tomaron muestras no alteradas en anillos de volumen conocido de cada horizonte diagnóstico presente $(\mathrm{O}$, Ao y $\mathrm{Bw})$, para determinar la densidad aparente, textura, curvas de retención de humedad, contenido de materia orgánica, conductividad hidráulica saturada y la densidad de distribución de las raíces finas, en el perfil del suelo.

Análisis de datos. Con los datos medidos durante dos años, se calculó la evapotranspiración de referencia, empleando la ecuación de Penman-Moteith, empleando la ecuación:

$$
\lambda \mathrm{E}=\frac{\Delta \mathrm{R}_{\mathrm{n}}+\rho \operatorname{Cp}\left(\mathrm{e}_{\mathrm{s}}-\mathrm{e}_{\mathrm{a}}\right) / \mathrm{r}_{\mathrm{a}}}{\Delta+\gamma\left(1+\mathrm{r}_{\mathrm{s}} / \mathrm{r}_{\mathrm{a}}\right)}
$$


Donde,

$\mathrm{l}=$ Calor latente de vaporización del agua $(\mathrm{J} / \mathrm{kg})$

$\mathrm{D}=$ Pendiente de la curva de saturación de vapor de presión (mbar/K)

$\mathrm{Rn}=$ Radiación solar neta $\left(\mathrm{W} / \mathrm{m}^{2}\right)$

$\mathrm{r}=$ Densidad del aire $\left(\mathrm{kg} / \mathrm{m}^{3}\right)$

$\mathrm{Cp}=$ Calor específico del aire a presión constante (J/kg.K)

es $=$ Presión de vapor a punto de saturación (mbar)

ea $=$ Presión de vapor actual (mbar)

$\mathrm{ra}=$ Resistencia aerodinámica $(\mathrm{s} / \mathrm{m})$

$\mathrm{g}=$ Constante psicrométrica $(\mathrm{mbar} / \mathrm{K})$

$\mathrm{rs}=$ Resistencia estomatal $(\mathrm{s} / \mathrm{m})$

Desde la perspectiva hidrológica, una de las características más llamativas de los ecosistemas de páramo es su alto rendimiento hídrico, entendido como la proporción de precipitación (P), que se convierte en caudal $(\mathrm{Q})$ y que se estima como Q/P. En este caso, se evalúo el rendimiento hídrico mensual y promedio para cada páramo.

Otro elemento relevante de los páramos es su regulación hídrica, entendida como la velocidad y la magnitud de la respuesta hidrológica de una cuenca ante eventos de precipitación y la conservación del caudal de estiaje, en los períodos de verano. Para analizar esta característica, se empleó el método de separación de caudales de línea recta entre los puntos de inflexión del hidrograma, con el fin de diferenciar el caudal base y el caudal de respuesta inmediata de las cuencas, a los eventos de precipitación. Para evaluar la respuesta de las cuencas a eventos de precipitación durante las distintas épocas del año, en cada una, se seleccionaron tres eventos representativos de un período de lluvias, un período seco y de la transición. Asimismo, se generaron las curvas de excedencia de caudales para el período medido, estimadas como la diferencia entre la unidad y la probabilidad de ocurrencia de un caudal dado (1-P, en mm.día $\left.{ }^{-1}\right)$ y que, al ser expresado en lámina de agua, incorpora el área de captación, permite comparaciones entre ellas.

\section{RESULTADOS Y DISCUSIÓN}

Características climáticas de los páramos. La tabla 1 presenta los resultados de dos años de mediciones hidroclimáticas en los tres páramos en Colombia, incluyendo los cálculos de evapotranspiración de referencia (ETo), así como la localización y la altitud de las estaciones meteorológicas. Además de los promedios, se muestran los valores extremos, que dan idea de la amplitud de los rangos que presentan estas variables. Las diferencias en las condiciones climáticas de los tres sitios, se relacionan con la altitud, la exposición a las masas de aire que ascienden desde los valles interandinos y las características de los ecosistemas circundantes. Se observa que Romerales, al ser el páramo con mayor altitud, tiene también las menores temperaturas, menor radiación solar y fuertes vientos, todo lo opuesto a las características que presenta Belmira, páramo de menor altitud. La radiación solar, en este caso, tiene una estrecha relación con la presencia de nubes bajas y de niebla (Bruijnzeel et al. 2011; Mulligan, 2010). El movimiento de las masas de aire durante el día juega un papel importante en las condiciones, en el funcionamiento y en la preservación de los ecosistemas de alta montaña tropical, ya que transporta cantidades significativas de vapor de agua desde zonas bajas hacia mayores altitudes, incrementando la humedad relativa en ambientes de alta montaña y formando niebla y nubes bajas (Ruiz et al. 2012). Lo anterior explica también los resultados obtenidos de ETo: Chingaza, al ser más húmedo, con lluvias y niebla constantes (Cárdenas et al. 2017), tiene valores bajos de salida de agua por este concepto, contrario a lo que se observa para Belmira, que no solo es el páramo de menor altitud, sino que además está situado en la cima de la montaña y no cuenta con barreras que intercepten las masas de aire húmedo ascendente.

La exposición de los páramos estudiados, por su parte, determina la cantidad de precipitación que reciben: Chingaza sobresale, por ser un páramo muy húmedo, con precipitaciones anuales de más de $3.000 \mathrm{~mm}$, mientras que Romerales registra valores de precipitación anual por debajo de $1.000 \mathrm{~mm}$. Chingaza, además, muestra la tendencia monomodal en la distribución temporal de la precipitación, como consecuencia de la influencia de la cuenca del río Orinoco, mientras que los otros dos páramos presentan tendencias bimodales en su distribución anual de lluvias (Figura 2).

Propiedades hidro-físicas de los suelos. Los suelos de los páramos estudiados, se caracterizan por tener altos contenidos de materia orgánica, generalmente, con presencia de cenizas volcánicas, baja densidad aparente, alta conductividad hidráulica saturada y alta capacidad de retención de humedad, aún a punto de marchitez permanente (Tabla 2). Lo anterior está de acuerdo con lo reportado por diferentes autores para suelos de páramo (Buytaert, 2004; Hincapié \& Tobón, 2011; Iñiguez et al. 2015; Mena et al. 2000).

El páramo de Romerales presenta suelos más profundos, pero con propiedades muy similares a las encontradas en Belmira, que tiene suelos poco profundos, mientras que el páramo de Chingaza exhibe las propiedades más características de suelos bien conservados, incluyendo la presencia de un horizonte orgánico $(\mathrm{O})$. Por el contrario, el deterioro en algunas de las propiedades hidro-físicas de los suelos de Belmira y Romerales está asociado con el pisoteo del ganado y con la remoción de material vegetal por quemas, cultivos o minería -como ocurrió en Belmira-.

Belmira, a pesar haber estado sometido a diferentes procesos de alteración de coberturas y de suelos, incluyendo re- 
Tabla 1. Valores anuales promedio de dos años de mediciones (2014-2016), de variables hidro-meteorológicas en los tres páramos.

\begin{tabular}{|c|c|c|c|c|c|c|c|c|c|c|c|c|c|c|c|}
\hline \multirow[t]{2}{*}{ Páramo } & \multirow{2}{*}{$\begin{array}{l}\text { Latitud } \\
\text { (N) }\end{array}$} & \multirow{2}{*}{$\begin{array}{l}\text { Longitud } \\
\text { (W) }\end{array}$} & \multirow{2}{*}{$\begin{array}{c}\text { Altitud } \\
(\mathrm{m})\end{array}$} & \multicolumn{3}{|c|}{ Temperatura $\left({ }^{\circ} \mathrm{C}\right)$} & \multicolumn{2}{|c|}{$\begin{array}{l}\text { Humedad } \\
\text { relativa (\%) }\end{array}$} & \multicolumn{2}{|c|}{$\begin{array}{l}\text { Radiación solar } \\
(\text { W.m-2 })\end{array}$} & \multicolumn{2}{|c|}{$\begin{array}{c}\text { Velocidad } \\
\text { viento }\left(\mathrm{m} \cdot \mathrm{s}^{-1}\right)\end{array}$} & \multirow{2}{*}{$\begin{array}{l}\text { Caudal } \\
(\mathrm{mm})\end{array}$} & \multirow{2}{*}{$\begin{array}{l}\text { ETa } \\
(\mathrm{mm})\end{array}$} & \multirow{2}{*}{$\begin{array}{l}\text { Precipi- } \\
\text { tación } \\
(\mathrm{mm})\end{array}$} \\
\hline & & & & Media & Max & Min & Media & Min & Media & Max & Media & Max & & & \\
\hline Belmira & $6^{\circ} 39.67^{\prime}$ & $75^{\circ} 40.38^{\prime}$ & 3118 & 10,4 & 18,8 & 4,0 & 91,9 & 36,0 & 328 & .487. & 2,9 & 10,7 & 886,1 & 434 & $.478,4$ \\
\hline Chingaza & $4^{\circ} 45.96^{\prime}$ & $73^{\circ} 49.63^{\prime}$ & 3570 & 11,3 & 26,7 & 0,8 & 85,0 & 25,0 & 271 & 1.019 & 3,4 & 18,1 & 1.979 & 245 & $3.098,1$ \\
\hline Romerales & $4^{\circ} 40.25^{\prime}$ & $75^{\circ} 25.15^{\prime}$ & 3840 & 5,3 & 13,5 & 0,0 & 90,7 & 13,4 & 257 & 1.390 & 3,8 & 16,6 & 380 & 273 & 969,7 \\
\hline
\end{tabular}
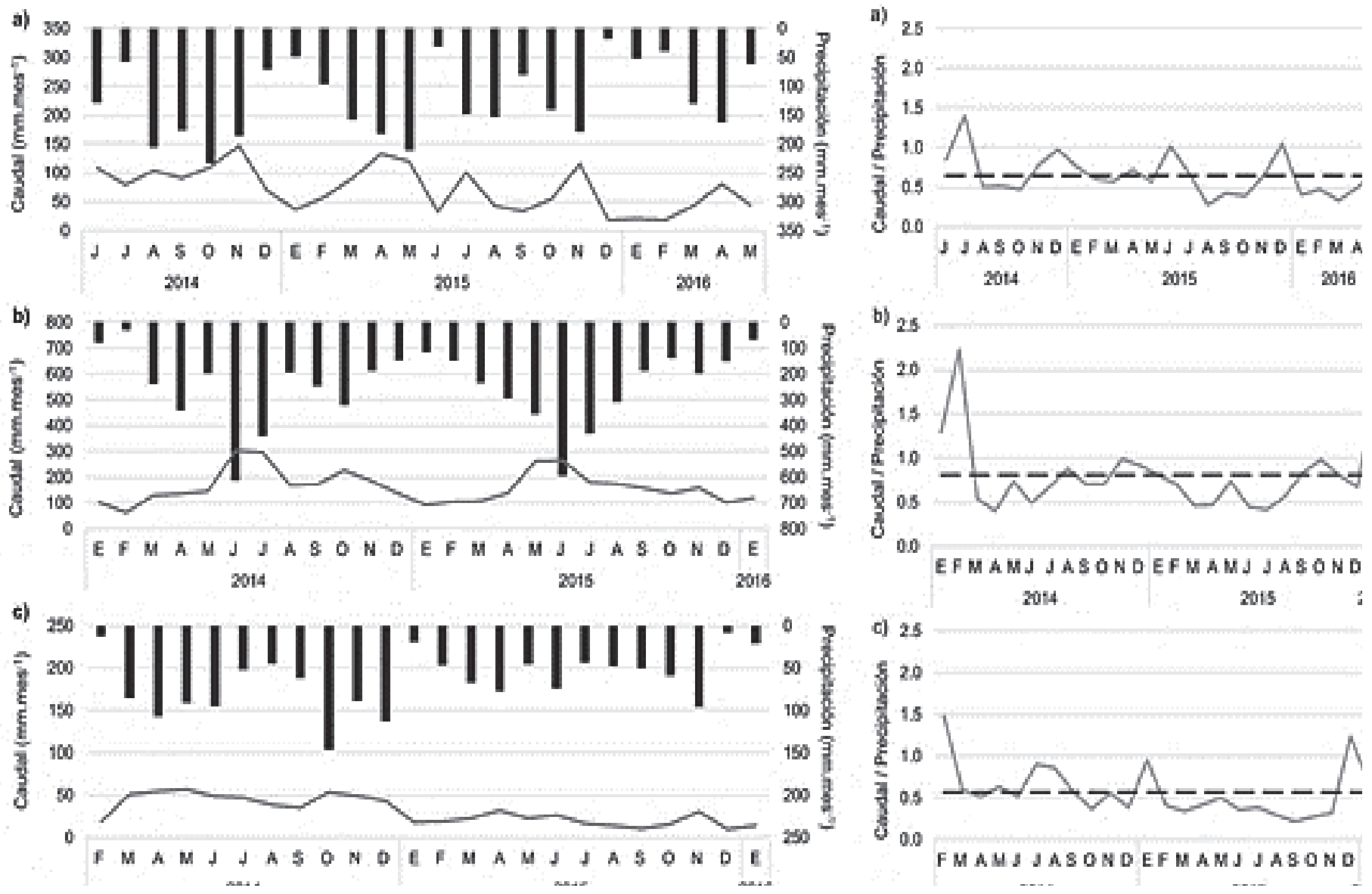

Figura 2. Precipitación y caudal mensual medidos (izq.) y rendimiento hídrico mensual (Q/P) mensual y promedio (línea punteada) en: a) Belmira, b) Chingaza y c) Romerales, para el período estudiado. 
Tabla 2. Valores promedio de las propiedades hidro-físicas de los suelos, para el perfil del suelo (horizontes A, B y O cuando estaba presente).

\begin{tabular}{|c|c|c|c|c|c|c|c|c|c|c|c|c|c|c|}
\hline \multirow{2}{*}{ Páramo } & \multirow{2}{*}{$\begin{array}{c}\text { Profun- } \\
\text { didad } \\
\text { del } \\
\text { suelo } \\
\text { (cm) }\end{array}$} & \multicolumn{3}{|c|}{$\begin{array}{c}\text { Contenido de Materia } \\
\text { Orgánica (\%) }\end{array}$} & \multicolumn{3}{|c|}{$\begin{array}{c}\text { Densidad aparente } \\
\left(\mathrm{g} \cdot \mathrm{cm}^{-3}\right)\end{array}$} & \multirow{2}{*}{$\begin{array}{l}\text { Tex- } \\
\text { tura }\end{array}$} & \multirow{2}{*}{$\begin{array}{l}\text { Ks media } \\
\left(\text { m.día }^{-1}\right)\end{array}$} & \multirow{2}{*}{$\begin{array}{l}\text { Densi- } \\
\text { dad de } \\
\text { raíces } \\
\left(\mathrm{g} \cdot \mathrm{cm}^{-3}\right)\end{array}$} & \multirow{2}{*}{$\begin{array}{c}\text { Hori- } \\
\text { zonte } \\
\text { O }\end{array}$} & \multirow{2}{*}{ Sat* } & \multirow{2}{*}{$\mathrm{CC}$} & \multirow{2}{*}{ PMP } \\
\hline & & Media & Max & Min & Media & Max & Min & & & & & & & \\
\hline Belmira & 41,4 & 14,10 & 27,84 & 6,78 & 0,63 & 0,84 & 0,36 & $\begin{array}{c}\text { arenosa } \\
\text { - franco } \\
\text { arenosa }\end{array}$ & 0,072 & 0,0148 & Ausente & SD & 0,60 & 0,20 \\
\hline $\begin{array}{l}\text { Chin- } \\
\text { gaza }\end{array}$ & 47,9 & 26,26 & 42,55 & 12,77 & 0,43 & 0,67 & 0,30 & franca & 1,694 & SD & $\begin{array}{l}\text { Pre- } \\
\text { sente }\end{array}$ & 0,61 & 0,56 & 0,33 \\
\hline $\begin{array}{l}\text { Rome- } \\
\text { rales }\end{array}$ & 71,7 & 14,60 & 24,50 & 1,35 & 0,61 & 0,71 & 0,48 & $\begin{array}{c}\text { franco } \\
\text { arenosa }\end{array}$ & 0,035 & 0,0026 & Ausente & 0,60 & 0,55 & 0,29 \\
\hline
\end{tabular}

*Sat: Contenido de humedad del suelo a saturación (10 KPa).

CC: Contenido de humedad del suelo a capacidad de campo $(33 \mathrm{KPa})$.

PMP: Contenido de humedad del suelo en punto de marchitez permanente $(1500 \mathrm{KPa})$.

SD: Sin dato.

moción de capas del suelo, que explican que presente suelos superficiales y poco desarrollados, ha estado bajo un proceso de restauración pasiva desde 1989 , previo a su declaratoria como Distrito de Manejo Integrado (en 2007), que ha permitido que los suelos hayan recuperado sus propiedades, a niveles similares a las que presentan otros páramos, medianamente intervenidos, como el de Romerales. Más aún, para algunos de los parámetros evaluados (Tabla 2), Belmira presenta valores superiores a los de Romerales, como conductividad hidráulica saturada, densidad de raíces y la cantidad de agua disponible para las plantas (CC-PMP) que, incluso, es mayor que la del páramo de Chingaza.

Por otro lado, aunque las condiciones climáticas que prevalecen en la alta montaña del trópico son limitantes ambientales que restringen el crecimiento de las especies, es notable la velocidad de regeneración y el crecimiento que tienen las plantas adaptadas a dichos ambientes, por ejemplo, luego de eventos nocivos, como incendios (observaciones de los autores), lo cual, es contrario a la idea generalizada de que los procesos en estos ecosistemas y su vegetación son exageradamente lentos y que, por tanto, su restauración tomaría muchas décadas.

Rendimiento hídrico en los páramos. La figura 2 presenta el comportamiento de la precipitación y el caudal mensual, medidos en cada cuenca durante dos años y el rendimiento hídrico $(\mathrm{Q} / \mathrm{P})$ mensual y promedio para cada páramo, durante el período estudiado. Las magnitudes de los caudales, como salidas de agua del sistema, guardan proporción con las magnitudes de las entradas, aunque las relaciones mues- tran diferencias: Belmira tiene, en promedio, un rendimiento hídrico de 0,60, mientras que en Chingaza es de 0,64 y en Romerales, de 0,39.

En Chingaza, el resultado obtenido se explica por la alta frecuencia de eventos de precipitación durante todo el año, por la menor radiación y por la permanencia de la humedad del suelo cercana a saturación, relacionada con los altos contenidos de materia orgánica y una densidad aparente muy baja. Bajo condiciones de saturación de humedad del suelo, la vegetación transpira menos, aunque las condiciones atmosféricas sean óptimas para inducir este proceso (Bruijnzeel et al. 2011), lo cual, determina bajas pérdidas de agua del sistema, a través de evapotranspiración (Tabla 1) y, por ende, un mayor rendimiento hídrico. Por el contrario, aun cuando el páramo de Romerales presenta una menor radiación solar, al estar a mayor altitud, tiene mayor exposición a vientos fuertes; además, sus suelos tienen menor contenido de materia orgánica comparado con el promedio de los suelos de páramos, lo que hace que la humedad de los suelos, aunque es alta, permanece por debajo de capacidad de campo. Esto último, sumado a los fuertes vientos, resulta en mayores pérdidas de agua por evapotranspiración.

De acuerdo con lo anterior, el páramo de Belmira presenta un rendimiento hídrico promedio mayor que Romerales y cercano a los valores reportados para otros páramos en Colombia (Tobón, 2009).

Regulación hídrica. La separación de los caudales en las tres cuencas dio como resultado que Belmira tiene la me- 
nor proporción de caudal base con respecto al caudal total $(61,7 \%)$, seguido por Chingaza, con $80,7 \%$, mientras que Romerales presenta la mayor contribución del caudal base al caudal total $(89,5 \%)$. Este comportamiento es coincidente con las curvas de excedencia de caudales (Figura 3), que resumen las respuestas de las cuencas estudiadas durante dos años. Las curvas de excedencia de caudales muestran que durante todo el período estudiado, en términos de lámina de caudal diario, Chingaza tiene los valores más altos, mientras que Romerales registra los más bajos, pero, además, este último evidencia mayores reducciones en su caudal durante los períodos de estiaje, como se ve en el recuadro del percentil 95, de la figura 3. También señala la figura 3, los picos de respuesta de los caudales en los percentiles más bajos: Chingaza y Belmira, por ejemplo, tienen los picos más altos; no obstante, proporcionalmente, el primero se triplica con respecto a su caudal medio, pero para el caso de Belmira, los caudales picos multiplican por 7 los caudales medios, lo que quiere decir que Belmira presenta menor regulación de sus caudales picos, lo cual, se explica por el tamaño de la cuenca -es la más pequeña-, pero, especialmente, por la menor profundidad de sus suelos. Otra característica a resaltar en las curvas de excedencia de caudales (Figura 3) es el cambio de la pendiente, ya que los cambios en las curvas de Romerales y de Chingaza son abruptos y ocurren en porcentajes bajos (4,1 y 10,2\%, respectivamente), mientras que para Belmira, no se evidencia un punto de quiebre, sino que la curva desciende suavemente, aunque se puede notar un cambio alrededor del $12,8 \%$, lo que significa que existen diferencias en el comportamiento del caudal, entre estos páramos. De lo anterior se puede concluir, que el páramo de Chingaza tiene una adecuada regulación hidrológica, aunque presenta caudales pico, que se explican por la saturación de sus suelos; Romerales, en cambio, tiene una excelente regulación de caudales de tormenta, pero el agua retenida en los suelos parece no aportar suficiente al caudal base, que se reduce notablemente durante los períodos de sequía; Belmira, por su parte, se encuentra en una situación intermedia, puesto que los caudales pico que presenta se relacionan, principalmente, con la menor profundidad del suelo y tiene baja proporción en las curvas de caudales.

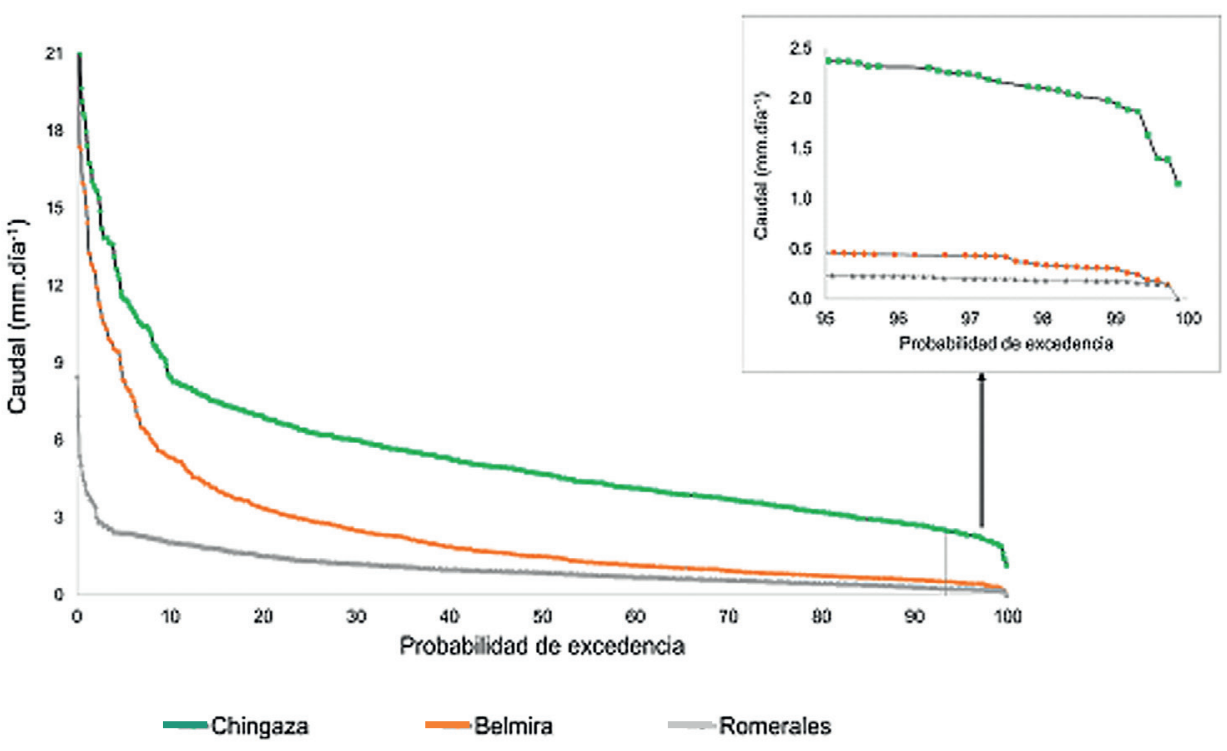

Figura 3. Curvas de probabilidad de excedencia de caudales para los tres sitios estudiados. El recuadro muestra el detalle de las curvas en el último $5 \%$.

Asimismo, un análisis de la respuesta de las cuencas a los eventos de precipitación (Figura 4) indica que, en términos generales, los páramos de Belmira y de Chingaza presentan tiempos de retardo similares (menores a una hora). Romerales, en cambio, muestra las respuestas en el caudal unas dos horas después de los eventos de precipitación. Lo anterior está relacionado con características propias de cada cuenca, principalmente su morfometría y el contenido de humedad de los suelos: la cuenca estudiada en Belmira tiene una menor área, forma redondeada y suelos superficiales. Chingaza presenta suelos cercanos a saturación durante la mayor parte del año. Romerales, por su parte, es la cuenca más 
extensa y tiene los suelos más profundos, con contenidos de humedad por debajo de capacidad de campo, gran parte del tiempo.
Conclusiones generales. Del presente estudio, se puede concluir que Belmira es un buen ejemplo de cómo páramos alterados, pueden recuperar gran parte de sus características y de su funcionamiento hidrológico en pocas décadas, bajo
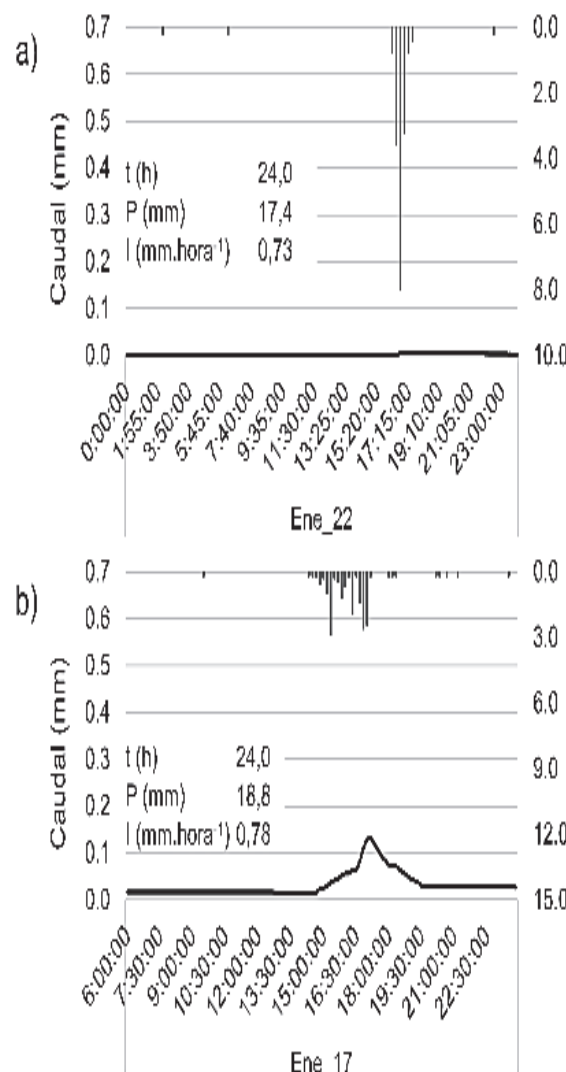

Ene 17

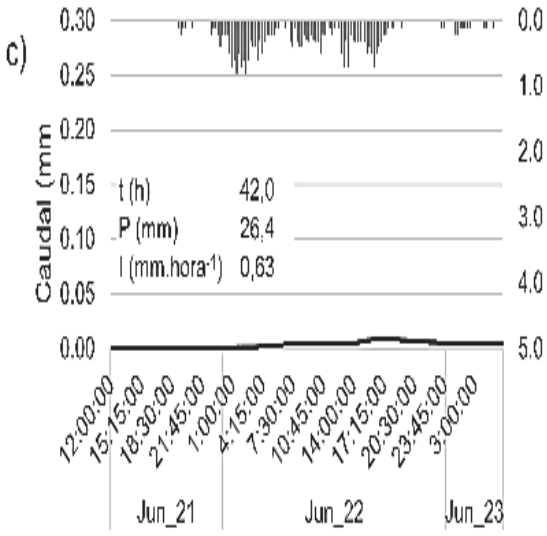

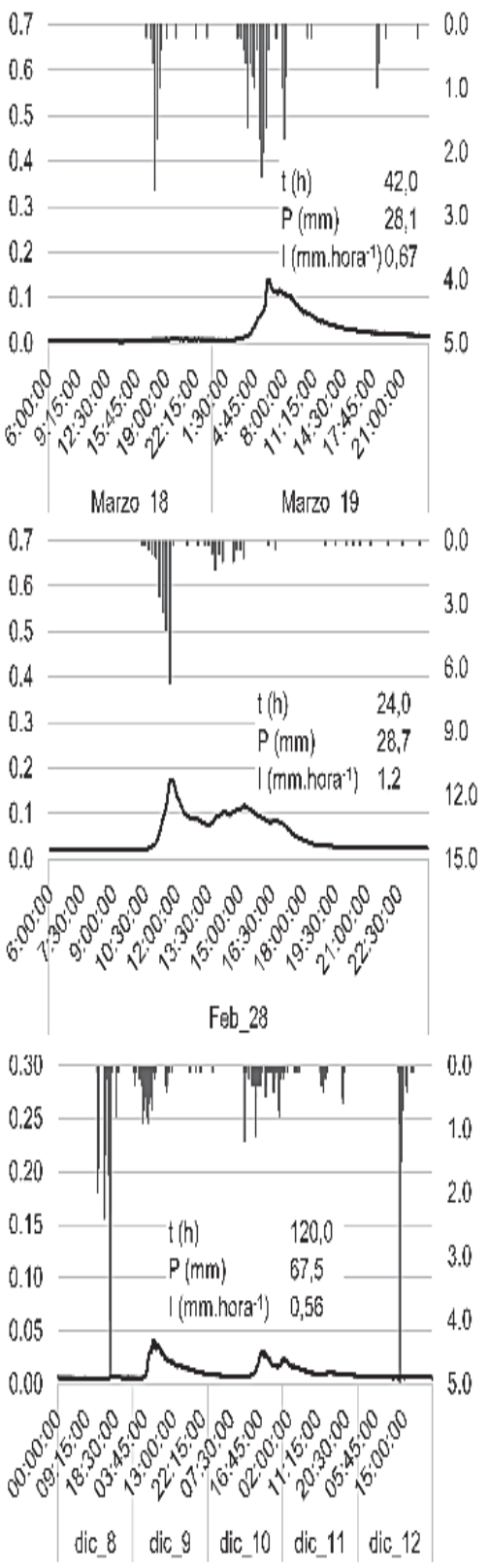

Figura 4. Eventos de lluvias y las respectivas respuestas de los caudales durante períodos de sequía, transición y períodos húmedos para: a) Belmira, b) Chingaza y c) Romerales. Los números en el recuadro señalan la duración del período seleccionado ( $\mathrm{t}$ en horas), la precipitación total registrada en dicho período ( $\mathrm{P}$ en $\mathrm{mm}$ ) y la intensidad promedia (I en mm.hora ${ }^{-1}$ ), para cada uno de los eventos seleccionados. 
un programa de protección. Específicamente, las propiedades hidrofísicas del suelo, el contenido de materia orgánica y el agua disponible para las plantas, se encuentran dentro de los rangos reportados para otros páramos y facilitan el establecimiento y el desarrollo de la vegetación, al no tener restricciones hídricas, para sus procesos fisiológicos. A su vez, las coberturas vegetales contribuyen a mejorar las condiciones biofísicas de los suelos.

En conjunto, los hallazgos reportados en este documento señalan que los páramos alterados pueden restaurar sus funciones hidrológicas en pocas décadas, incluso, bajo esquemas de restauración pasiva.

Agradecimientos: A Colciencias, por financiar el último año de estudios de María F. Cárdenas, a través de la convocatoria 727 - 2015 para doctorados nacionales y a la Facultad de Minas de la Universidad Nacional de Colombia, sede Medellín, por su apoyo financiero, administrativo y académico a María F. Cárdenas, durante su formación doctoral. Conflictos de intereses: El manuscrito fue preparado y revisado con la participación de todos los autores, quienes declaramos que no existe conflicto de intereses que ponga en riesgo la validez de los resultados presentados. Financiación: Este estudio fue financiado por Colciencias, a través del proyecto de investigación "Estudio ecohidrológicos de los páramos y los bosques alto-andinos, naturales e intervenidos: Análisis de la vulnerabilidad y adaptabilidad al cambio climático", en la convocatoria CT\&i 569-2012.

\section{BIBLIOGRAFÍA}

1. BECKER, A. 2005. Runoff processes in mountain headwater catchments: Recent understanding and research challenges. In: Huber, U.M.; Bugmann, H.K.M.; Reasoner, M.A. (Eds.). Global Change and Mountain Regions: An Overview of Current Knowledge. Springer, Dordrecht, The Netherlands. p.283295.

2. BRUIJNZEEL, L.A.; MULLIGAN, M.; SCATENA, F.N. 2011. Hydrometeorology of tropical montane cloud forests: emerging patterns. Hydrol. Process. 25:465498.

3. BUYTAERT, W. 2004. The properties of the soils of the south Ecuadorian paramo and the impact of land use changes on their hydrology. Catholic University Leuven, Leuven, Belgium. 205p.

4. BUYTAERT, W.; CÉLLERI, R.; DE BIÈVRE, B.; CISNEROS, F.; WYSEURE, G.; DECKERS, J.; HOFSTEDE, R. 2006a. Human impact on the hydrology of the Andean páramos. Earth-Science Rev. 79:53-72.
5. BUYTAERT, W.; DECKERS, J.; WYSEURE, G. 2006b. Regional variability of volcanic ash soils in south Ecuador: the relation with parent material, climate and land use. Catena. 70:143-154.

6. BUYTAERT, W.; DECKERS, J.; WYSEURE, G. 2006c. Description and classification of nonallophanic Andosols in south Ecuadorian alpine grasslands (paramo). Geomorphology. 73(3-4):207-221.

7. CÁRDENAS, M.F.; TOBÓN, C.; BUYTAERT, W. 2017. Contribution of occult precipitation to the water balance of páramo ecosystems in the Colombian Andes. Hydrological Processes. p.1-10.

8. CÉLLERI, R.; FEYEN, J. 2009. The hydrology of tropical Andean ecosystems: Importance, knowledge status, and perspectives. Mt. Res. Dev. 29:350-355.

9. CORPORACIÓN AUTÓNOMA REGIONAL DEL CENTRO DE ANTIOQUIA -CORANTIOQUIA-. 1999. Páramo encuentro de Regiones. Proyecto "Conservación, Ordenamiento y Manejo del Sistema de Páramos y Bosques Altoandinos del Noroccidente Medio Antioqueño," Medellín: Corantioquia. 134p.

10. DIAZ, H.F.; GROSJEAN, M.; GRAUMLICH, L. 2003. Climate variability and change in high elevation regions: Past, present \& future. Clim. Change. 59:1-4.

11. FARLEY, K.; KELLY, E.; HOFSTEDE, R. 2004. Soil organic carbon and water retention after conversion of grasslands to pine plantations in the Ecuadorian Andes. Ecosystems. 7:729-739.

12. GOLDSMITH, G.R.; MUÑOZ-VILLERS, L.E.; HOLWERDA, F.; MCDONNELL, J.J.; ASBJORNSEN, H.; DAWSON, T.E. 2012. Stable isotopes reveal linkages among ecohydrological processes in a seasonally dry tropical montane cloud forest. Ecohydrology. 5:779-790.

13. GUZMÁN, P.; BATELAAN, O.; HUYSMANS, M.; WYSEURE, G. 2015. Comparative analysis of baseflow characteristics of two Andean catchments, Ecuador. Hydrol. Process. 29:3051-3064.

14. HERZOG, S.K.; MARTÍNEZ, R.; JØRGENSEN, P.M.; TIESSEN, H. (Eds.). 2012. Cambio Climático y Biodiversidad en los Andes Tropicales. Sao José dos Campos, y Comité Científico sobre Problemas del medio Ambiente (SCOPE), París. 410p. 
15. HINCAPIÉ, E.; TOBÓN, C. 2011. Caracterización de las propiedades hidrofísicas de los andisoles en condiciones de ladera. Rev. Suelos Ecuatoriales. 3:67-78.

16. HOFSTEDE, R.; SEGARRA, P.; MENA, P. (Eds.). 2003. Los páramos del Mundo. Proyecto Atlas Mundial de Los Páramos. Global Peatland Initiative/ NC-IUCN/ EcoCiencia, Quito. 300p.

17. IÑIGUEZ, V.; MORALES, O.; CISNEROS, F.; BAUWENS, W.; WYSEURE, G. 2015. Analysis of the drought resilience of Andosols on southern Ecuadorian Andean páramos. Hydrol. Earth Syst. Sci. Discuss. 12:11449-11484.

18. LORA-GÓMEZ, C.A. 2005. Proceso de Formulación e Implementación del Plan de Manejo del Parque Nacional Natural Chingaza, Bogotá. 234p.

19. MENA, P.; JOSSE, C.; MEDINA, G. (Eds.). 2000. Los Suelos del Páramo. GTP/Abya Yala, Quito. 75p.

20. MORALES, M.; OTERO, J.; VAN DER HAMMEN, T.; TORRES, A.; CADENA, C.; PEDRAZA, C.; RODRÍGUEZ, N.; FRANCO, C.; BETANCOURTH, J.C.; OLAYA, E.; POSADA, E.; CÁRDENAS, L. 2007. Atlas de páramos de Colombia. Instituto de Investigación de Recursos Biológicos Alexander von Humboldt, Bogotá. 208p.

21. MOSQUERA, G.M.; LAZO, P.X.; CÉLLERI, R.; WILCOX, B.P.; CRESPO, P. 2015. Runoff from tropical alpine grasslands increases with areal extent of wetlands. Catena. 125:120-128.

22. MULLIGAN, M. 2010. Modelling the tropics-wide extent and distribution of cloud forests and cloud forest loss with implications for their conservation priority. In: Bruijnzeel, L.; Scatena, F.N.; Hamilton, L. (Eds.). Tropical Montane Cloud Forests: Science for Conservation and Management. Cambridge University Press, Cambridge. p.14-38.

23. OCHOA-TOCACHI, B.F.; BUYTAERT, W.; DE BIÈVRE, B.; CÉLLERI, R.; CRESPO, P.; VILLACÍS, M.; LLERENA, C.A.; ACOSTA, L.; VILLAZÓN, M.; GUALLPA, M.; GIL-RÍOS, J.; FUENTES, P.; OLAYA, D.; VIÑAS, P.; ROJAS, G.; ARIAS, S. 2016. Impacts of land use on the hydrological response of tropical Andean catchments. Hydrol. Process. 30(22):4074-4089.

24. RUIZ, D.; MARTINSON, D.G.; VERGARA, W. 2012. Trends, stability and stress in the Colombian Central Andes. Clim. Change 112:717-732.

25. RUIZ, D.; MORENO, H.A.; GUTIÉRREZ, M.E.; ZAPATA, P.A. 2008. Changing climate and endangered high mountain ecosystems in Colombia. Sci. Total Environ. 398(1-3):122-132.

26. SALENTO, 2000. Esquema de Ordenamiento Territorial EOT municipio de Salento, Quindío, Salento, Quindío: Consejo Municipal de Salento. 256p.

27. TOBÓN, C. 2009. Los bosques andinos y el agua, Serie Investigación y Sistematización \#4. Programa Regional ECOBONA-INTERCOOPERACIÓN, CONDESAN, Quito. 122p.

Recibido: Septiembre 13 de 2017

Aceptado: Noviembre 22 de 2017

Cómo citar:

Cárdenas, M.F.; Conrado Tobón, C. 2017. Recuperación del funcionamiento hidrológico de ecosistemas de páramo en Colombia. Rev. U.D.C.A Act. \& Div. Cient. 20(2): 403-412. 